\title{
Vinculación entre la Facultad de Ciencias e Ingenierías, el Estado y las instituciones privadas nicaragüenses
}

\section{Connection between the Faculty of Sciences and Engineering, the State and the nicaraguan private institutions}

\author{
Juan de Dios Bonilla Anduray ${ }^{1}$ \\ Manuel Enrique Pedroza ${ }^{2}$
}

\section{RESUMEN}

La comunidad universitaria representa en muchos países el punto de avance en la ciencia y la tecnología, es por ello, que las sociedades requieren un tipo de relación con las universidades. En nuestro país en los últimos años el contexto es favorable para llevar la ciencia al pueblo, la relación entre la Universidad y el Gobierno de Nicaragua se encuentra en las mejores condiciones ya que el Plan Nacional de Desarrollo Humano posee proyectos y políticas a favor del acercamiento y sinergia con las universidades. El presente ensayo muestra las acciones que la UNAN-Managua, en especial la Facultad de Ciencias e Ingenierías realiza para vincular el estado, la empresa privada y la universidad.

Palabras clave: Extensión Universitaria; Vinculación Universidad Estado; Modelo I+D+i de la UNANManagua.

\begin{abstract}
The university community represents in many countries the point of development in science and technology; therefore the societies require a kind of relationship with the universities. In Nicaragua in recent years, the context has been advantageous to bring science to the people, the relationship between the University and the Government of Nicaragua is in the best circumstances since the National Plan for Human Development has projects and policies in favor of the agreement and synergy with the universities. This essay displays the actions that UNAN- Managua through the Faculty of Sciences and Engineering performs in order to link the state, the private sector and the university.
\end{abstract}

Keywords: university extension; connection between the universities and the State; I+D+I model of the UNAN-Managua.

DOI: https://doi.org/10.5377/multiensayos.v3i6.9695

Recibido: 02 de noviembre de 2017

Aceptado: 04 de enero de 2018

1 Docente UNAN-Managua, Managua. Estudiante del Programa de Doctorado en "Gestión y Calidad de la Investigación Científica". UNAN Managua. Correo electrónico: jbanduray@hotmail.com

2 Profesor Titular de la UNAN-Managua. Coordinador del Programa de Doctorado en "Gestión y Calidad de la Investigación Científica". UNAN Managua. Correo electrónico: hppedroza@gmail.com 


\section{INTRODUCCIÓN}

Con el paso del tiempo se han ido produciendo una serie de revoluciones, la revolución agrícola, la revolución artesanal, la revolución industrial y post industrial y la actual revolución de la información y el conocimiento.

Esta última, surge principalmente cuando internet llega a la vida cotidiana de las personas, no se puede afirmar que esta sociedad está totalmente globalizada, ya que hay sitios donde todavía el acceso a internet no es masivo o es inexistente.

Estas condiciones de entorno hacen necesario un cambio en la forma de actuar de los individuos principalmente en su pensamiento que debe ser crítico, abstracto y sistémico, el modo de trabajo debe ser en equipo y gozar del conocimiento experimental.

En términos de educación, se dan avances debido a la influencia y uso de las denominadas Tecnologías de la Información y Comunicación (TICS), pero con ello, surge un reto enorme para las instituciones educativas que es el vínculo de los procesos académicos y los currículos a las necesidades y demandas del sector empresarial y la organización política vigente.

En este contexto, la extensión universitaria se inserta dentro de las estrategias de mayor prioridad en el encargo social de las universidades para responder a su misión de formadoras de los profesionales con un grado de cientificidad y humanismo que la sociedad nicaragüense espera para su desarrollo. Se puede afirmar entonces que la universidad tiene una marcada responsabilidad con la sociedad que se concreta en el actuar por el mejoramiento económico, social y cultural como sistema de vida del pueblo.

En lo particular la Universidad Nacional Autónoma de Nicaragua (UNAN-Managua) tiene el proceso de extensión universitaria como una función propia de la institución que contribuye directamente en el proceso formativo de los estudiantes de cada una de las carreras ofrecidas por dicha alma mater.

El plan estratégico de la UNAN Managua establece sobre el proceso de extensión lo siguiente: "Las funciones de proyección y extensión constituyen una responsabilidad social de la universidad para extender su acción educativa hacia la comunidad nacional y para fortalecer las relaciones de cooperación e intercambio"(UNAN Managua, 2015).

Entre los objetivos principales para el proceso de extensión en la UNAN-Managua se encuentran fortalecer el sistema de comunicación e información que posibilite el permanente intercambio de saberes y conocimientos con la sociedad y el estado, vincular la formación de los estudiantes al proceso productivo y a las necesidades objetivas del desarrollo económico en función de los intereses nacionales (UNAN Managua, 2015). 
Así mismo, el modelo de Investigación + Desarrollo + innovación de la UNAN-Managua contempla en su definición el vínculo entre la universidad y su entorno como es el estado y la empresa privada, interactuando de una forma cíclica y sistémica para el bienestar de la sociedad. El modelo $\mathrm{I}+\mathrm{D}+\mathrm{i}$ plantea que la extensión universitaria es el motor que impulsa el efecto de desbordamiento tecnológico, desde la universidad hacia la sociedad, es el medio institucional de difusión y adopción de conocimientos, que garantiza el apoyo y retroalimentación a la investigación, lo que permite el fortalecimiento de la vinculación Universidad-Estado, Universidad-Empresa, Universidad-Sociedad (Pedroza Pacheco, 2015).

\section{DESARROLLO}

La Facultad de Ciencias e Ingenierías, como parte de la Universidad Nacional Autónoma de Nicaragua (UNAN-Managua), realiza el proceso de extensión universitaria, en beneficio de la sociedad Nicaragüense, por tal razón, a continuación se detallan las acciones más relevantes de este proceso en cada uno de los departamentos que la conforman.

\section{Vinculo del Departamento de Computación con las Empresas Nicaragüenses}

Una primera actividad a destacar corresponde al desarrollo del proceso de innovación en el Ministerio de la Juventud (MINJUVE), que da lugar a varios programas de cooperación con la Facultad de Ciencias e Ingenierías y principalmente con el Departamento de Computación.

Existe una relación de sinergia entre la Oficina de Atención a Jóvenes Universitarios y Profesionales del MINJUVE y la Facultad de Ciencias de la UNAN-Managua. Esta estructura tiene como objetivo permitir a los jóvenes nicaragüenses integrarse a procesos de formación y capacitación continua, reconociendo sus intereses y aportando desde su participación en tareas de servicio, fomentando la investigación, innovación y tecnología como aporte a la comunidad.

En este contexto, el Departamento de Computación desarrolla el diplomado en Gestión de Tecnologías de Información e Innovación, bajo la dirección del departamento y cuyos protagonistas son jóvenes de diferentes áreas del conocimiento que fueron beneficiados por el programa de capacitación y formación juvenil, que dirige el Ministerio de la Juventud.

Este diplomado se inició el sábado 7 de mayo del 2016 con la asistencia de veintiséis estudiantes, de los cuales un $65.38 \%$ corresponden al género femenino y un $34.62 \%$ de varones, destacando la restitución del derecho a la educación por el gobierno actual y la respuesta pertinente de parte del sistema nacional de educación superior, en este caso el colectivo de docentes del Departamento de Computación. 
El diplomado anteriormente descrito presenta una promoción del 88\% y un índice de deserción del 12\%, considerándose un éxito rotundo en la aplicación de nuevas metodologías y técnicas didácticas en el proceso de enseñanza aprendizaje centrado en el alumno.

En esta práctica de cooperación, se creó un segundo diplomado denominado Gestión de Proyectos y Emprendimiento, el cual cuenta con treinta estudiantes profesionales de diversas áreas de la ciencia, además dicho programa académico tiene cobertura a nivel nacional y un sólido plan curricular que permite a los jóvenes desarrollar habilidades y capacidades en el desarrollo de su propia empresa y la administración de los proyectos respectivos.

Por otra parte, el Departamento de Computación responde a la demanda social en las comunidades que requieren desarrollo de polo económico, colaborando con la labor de la Fundación Raechel\& Jackie foundation cuya finalidad es mejorar el acceso, calidad y relevancia de la educación para la juventud en Centroamérica.

Un primer proyecto de cooperación con la fundación Raechel\& Jackie foundation corresponde a la elaboración del diagnóstico de condiciones tecnológicas para la creación de un laboratorio de computación en la zona rural del municipio de San Juan del Sur, el diagnostico consistió en verificar las condiciones Técnico-ambientales para la implementación de un pequeño centro de cómputo, que permitiera desarrollar los conocimientos tecnológicos a los jóvenes y maestros rurales.

Posteriormente se realizaron dos proyectos de capacitación con la cobertura de planta docente del departamento de Computación y la logística a cargo de la fundación Raechel\& Jackie foundation, de este proyecto resulto una promoción de cuarenta jóvenes de la comunidad dotados de los conocimientos básicos de computación que exigen los propietarios de los lugares turísticos para brindarles empleo.

Estos proyectos de capacitación lograron impactar el desarrollo académico de la comunidad de Tortuga e incidieron en la visión y modo de vida de la población joven que fue beneficiada con becas para asistir a estas capacitaciones.

Otras formas de vincular la academia con la sociedad son por medio de las prácticas profesionales de los estudiantes que cursan los últimos años de las carreras de ingeniería en computación y la ingeniería en sistemas de información, cuya contribución al desarrollo tecnológico de las pymes consiste en la creación de software elaborados con los criterios de calidad establecidos en las normas internacionales, esto representa un avance en el desarrollo intelectual del talento y la creatividad de los futuros profesionales del área de tecnología de la UNAN-Managua. 


\section{Vinculo del Departamento de Matemáticas con las Empresas Nicaragüenses}

Para el primer semestre del 2016, en conjunto a la Facultad de Humanidades y Ciencias Jurídica, los estudiantes de la Carrera de Ingeniería en Estadística formaron parte de un equipo, para la ejecución de la investigación: "Proyecto de Investigación: Un estudio local para el Desarrollo: Historia del Departamento de Rio San Juan y Sus Municipios”.

Para el segundo semestre se le brindo acompañamiento a la Facultad de Educación e Idiomas en los procesos de: Organización de la información, Digitación así como los respectivos análisis que cumplieron con los objetivos de la investigación: "Estrategias de Continuidad Educativa de Prescolar, Primero al Tercer Grado", ejecutada por el Ministerio de Educación, Dirección General de Educación Primaria(Parrales Cano, 2017).

Enfocados en las principales Líneas de Investigación orientadas al fortalecimiento interno de UNANManagua y las Líneas de Investigación orientadas en el PNDH, de la líneas de Investigación de la maestría en Métodos de Investigación Científica. Del inciso 2. Seguridad y Soberanía Alimentaria y Nutricional SSAN.

Se logró impulsar la aprobación de la investigación a cargo del Lic. Henrry Luis López García, titulada: "Evaluación del estado actual de la Soberanía y Seguridad Alimentaria y Nutricional de los Municipio: Masatepe, Nandasmo, Niquinohomo, Catarina y San Juan de Oriente del departamento de Masaya, en el periodo del año 2016"(Parrales Cano, 2017).

\section{Vinculo del Departamento de Biología con las Empresas Nicaragüenses}

El departamento de Biología, mantiene relaciones estrechas con las Alcaldías Municipales con las que realiza programas en conjunto sobre el tema de educación ambiental. Los docentes y estudiantes realizan un acompañamiento a la administración del Parque Nacional Volcán Masaya en el estudio de especies existentes en la reserva Volcán Masaya.

Se mantiene una alianza estratégica con el MARENA para la elaboración de estudios a profundidad sobre inventarios de especies de flora y fauna nacional en beneficio de actualizar los datos que dicho ministerio gestiona. El zoo criadero de reptiles colabora con la formación de estudiantes de secundaria de los diferentes centros de Nicaragua con el objetivo de incentivar la preservación de la especie iguana, iguana.

Otro punto relevante es la participación del departamento de Biología como miembro activo de la Red Mesoamericana de Investigación y Desarrollo de Biocombustibles cuyo objetivo es "Fomentar la investigación y transferencia de tecnología, y promover el intercambio de experiencias, recursos humanos y mejores prácticas entre los países del Proyecto Mesoamérica, a fin de asegurar un 
crecimiento ordenado del sector de los biocombustibles en la región mesoamericana"(Red mesoamericana de investigación y desarrollo de biocombustibles, 2016).

\section{Vinculo de la Escuela de Física con las Empresas Nicaragüenses}

La escuela de Física realiza su proceso de extensión por medio del observatorio astronómico. El observatorio posee una alta vinculación con el MINED y la Unión Internacional de Astronomía (IAU), por tal razón, se realiza un curso anual con duración de cuatro días cuyos protagonistas son los docentes de primaria y la cobertura del evento es a nivel nacional. Este curso tiene como objetivo dotar de habilidades y capacidades en la aplicación práctica de la astronomía en las ciencias naturales, se hace uso de herramientas didácticas actuales.

Además, una de las actividades cotidianas en el observatorio es recibir a lo largo del año en las instalaciones, la visita de cientos de estudiantes de educación secundaria, los cuales reciben asesoría en el uso del telescopio y charlas sobre temas especializados en la Astronomía por parte del personal, por ejemplo la relación de tamaño, temperatura y color de estrellas, así como otros temas de Astrofísica.

Actualmente, el observatorio está realizando el primer Seminario de Astronomía Cultural Nicaragüense: "Entre Volcanes y Estrellas", este seminario tiene como misión el proveer al participante de las competencias necesarias para identificar, investigar, comprender y difundir la relación existente entre la observación de fenómenos astronómicos y la influencia que éstos eventos ejercieron sobre la evolución cultural de los pueblos - en especial en el contexto mesoamericano -, utilizando para ello un enfoque interdisciplinario. Este enfoque debe contar con disciplinas y ciencias que aporten conocimientos, metodologías y puntos de vista para un análisis integral de las culturas que poblaron la región Mesoamérica.

La importancia de este taller, se denota en el hecho de que la Astronomía Cultural en América ha representado una herramienta valiosa en la interpretación cultural de los pueblos del pasado y constituye una herramienta igualmente importante para conocer a los pueblos indígenas, en especial de la región centroamericana.

Este conocimiento debe llevar a una inclusión social y un desarrollo sostenible de dichos pueblos y las sociedades y Etno astronomía, como sub disciplina de la Astronomía Cultural se torna de la máxima relevancia en la región Centroamericana. En especial en el territorio nicaragüense con un gran potencial cultural y una alta necesidad de cubrir el vacío de generación de conocimiento en este campo.

Finalmente, se está elaborando un proyecto sobre olimpiadas de Astronomía con el fin de promover interés en los estudiantes de cuarto y quinto año de secundaria en temas de Astronomía y ciencias espaciales, esta actividad se realizará con el apoyo del Ministerio de Educación. 


\section{Vínculo entre el Departamento de Construcción y las Empresas Nicaragüenses}

El Departamento de Construcción posee una alta vinculación con las Alcaldías Municipales, donde se realizan trabajos de Planificación de urbanizaciones, principalmente en levantamiento de datos in situ, ejemplo lotificaciones, calidad de viviendas, entre otros, que permiten a las municipalidades validar los datos que existen en la base de datos del Instituto Nacional de Desarrollo (INIDE).

El apoyo de los docentes y estudiantes a las municipalidades consiste en el trabajo realizado por los jóvenes que verifican y aportan más detalles que enriquecen la base de datos aportada por el INIDE para uso de las Alcaldías.

Así mismo, se participa en ferias vocacionales donde se muestran los resultados de los proyectos de beneficio social, entre ellos, la elaboración de proyectos habitacionales a bajo costo con aplicación de materiales agradables al medio ambiente, con sistemas constructivos tradicionales y ecológicos. También se elaboran anteproyectos reales de beneficio institucional para escuelas, centro de desarrollo infantil en la parte urbana.

Se han realizado proyectos a la Policía Nacional en anteproyectos de remodelación de instalaciones o infraestructura nuevas. Por otra parte, en colaboración con el desarrollo cultural del país, se desarrolló un proyecto de diseño Arquitectónico de un museo arqueológico ubicado en Santo Tomás, Chontales.

Por otra parte, se tienen relaciones con las Empresas privadas en la elaboración de diseños arquitectónicos, estos proyectos se han expuesto en ferias vocacionales con más aplicación de los conocimientos obtenidos en las asignaturas de Práctica I y II del Plan de Estudio de la Carrera de Arquitectura.

La carrera de Arquitectura ha contribuido también en el desarrollo de infraestructura de la UNANManagua, por ejemplo se realizó el diseño de los Kioscos tecnológicos para uso de los estudiantes del Recinto Rubén Darío, así mismo se contribuye en el Diseño de las estructuras metálicas del pabellón 68 de la UNAN. Otro aporte de parte de la carrera de Arquitectura fue la remodelación del Comedor Central de la UNAN-Managua.

Por otra parte, se desarrolló vinculación con otras universidades en el primer festival de cine "Ciudad y Arquitectura", ubicado en el Auditorio doce, los días 10 al 25 de Mayo del 2016, en esta actividad la participación en las ponencias por parte de los docentes de la carrera de Arquitectura fue notoria.

En dicha actividad participaron otras instituciones como la UAM, UNI, Guerrilla de comunicaciones, Raíces de Nicaragua, Federación Cristal, Casa de los tres mundos, Camila Films, COAM (Colegio 
de Arquitectos de Nicaragua, Asociación Nicaragüense de Ingenieros y Arquitectos, Coordinadora Nacional de Estudiantes de Arquitectura (CONEA).

El desarrollo del II Fotocamina Nicaragua 2016, se realizó en la Ciudad de León bajo el lema "Arte, Arquitectura y Patrimonio", el 20 de Mayo, con participación de las diferentes universidades y CONEA, así como invitados internacionales.

\section{Vínculo entre el Departamento de Química y las Empresas Nicaragüenses}

Dentro del proceso de extensión, uno de los logros más relevantes se encuentra la elaboración de una Maleta Pedagógica para la carrera de Química Farmacéutica con el financiamiento y acompañamiento de USAID, este proyecto involucró el desarrollo total de instrumentos y capacitaciones por el colectivo docente y especialistas en las áreas relacionadas a los contenidos y herramientas necesarias en el área, se ejecutó en el periodo del año 2010 al 2015(Gonzalez, 2017). Así mismo, se establece una relación cercana y de colaboración mutua con el MINSA para establecer las prácticas profesionales en las farmacias de los hospitales y Centros de Salud del sistema nacional de salud pública.

También, se crearon vínculos con hospitales, clínicas y farmacias privadas donde los estudiantes de la carrera de Química Farmacéutica realizan las funciones de prácticas en sus modalidades de familiarización, especialización y profesionalización, todo esto se lleva a cabo a lo largo y ancho del territorio nacional.

Se mantienen lazos de cooperación con todos los laboratorios e industrias, distribuidoras de productos farmacéuticos del País, en beneficio de las instituciones pertenecientes al sector encargado del desarrollo y distribución de productos farmacéuticos.

Otra contribución que cabe señalarse, corresponde a la participación en la elaboración de diagnósticos a la calidad de los alimentos de las diferentes empresas por medio del Laboratorio de Análisis Físico Químico de Alimentos, que pertenece a la Facultad de Ciencias e Ingenierías y dirige el departamento de Química.

Por su parte, los estudiantes de Química Industrial realizan prácticas en todas las Industrias ubicadas en los diferentes municipios del país y sectores diversos de la economía, como por ejemplo, en las empresas cementeras donde se analiza el proceso de elaboración del cemento, así como los componentes químicos que los conforman, también se participa en el área de alimentos donde se realizan proyectos con los mataderos, la cervecería, embutidos Delmor, industrias de licores, la coca cola, Pepsi cola entre otros.

Como parte del Departamento, los docentes y estudiantes de la carrera de Química ambiental hacen presencia en todos los laboratorios de agua, suelo y biotecnología, donde se visitan las 
comunidades y se verifican el tratamiento de residuos sólidos y líquidos así como deposición y tratamiento de aguas residuales por las empresas.

En apoyo a solicitudes de las Alcaldías, se realizan diagnósticos en conjunto y con apoyo del MARENA y MAGFOR con el fin de establecer el cumplimiento de normas y procedimientos adecuados en el tratamiento de los residuos.

Una acción positiva incluida en el modelo educativo actual, corresponde a la realización de las giras de campo en reservas y lagunas para realizar análisis de agua y suelos quepermiten medir los grados de contaminación presentes en las áreas bajo estudio, esto en coordinación con el MARENA.

Los docentes de prácticas con sus estudiantes realizan visitas a la finca modelo DISNAYA de la ciudad de Estelí, donde se elaboran Fitofármacos para conocer a fondo el proceso de elaboración y verificar el tratamiento que se aplica a dichos fármacos.

En el caso de Química Industrial se realizan varias giras de campo a las plantas de energía para observar y poner en práctica los conocimientos del proceso industrial, permitiendo esto realizar recomendaciones pertinentes.

Finalmente, cabe destacar la importancia que tiene la asignatura de Seminario de Formación Integral dentro de los planes de estudio, la cual permite el contacto con la sociedad, llegando a realizar actividades en beneficio de la salud y calidad de vida de la población.

\section{Vínculo entre el Departamento de Tecnología y las Empresas Nicaragüenses}

El Departamento de Tecnología es el último en formarse en la Facultad, su colectivo docente está formado principalmente por jóvenes.

Actualmente se están realizando gestiones para formalizar las relaciones cordiales con algunas instituciones privadas, las cuales se han realizado unos pocos proyectos de los estudiantes por medio de las prácticas profesionales de las carreras de ingeniería electrónica y la ingeniería industrial.

\section{CONCLUSIONES}

La relación entre la Universidad y el Gobierno de Nicaragua se encuentra en las mejores condiciones ya que el Plan Nacional de Desarrollo Humano posee proyectos y políticas a favor del acercamiento y sinergia con las universidades.

La UNAN-Managua consiente de la importancia del vínculo universidad, estado, empresa privada ha establecido un eje estratégico sobre la gestión de la proyección y la extensión universitaria. 
El desarrollo del modelo de gestión de la investigación, modelo $\mathrm{I}+\mathrm{D}+\mathrm{i}$ de la UNAN Managua promueve y define la relación entre la universidad y su entorno de forma clara, precisa y contundente. En esta línea de acción la Facultad de Ciencias e Ingenierías como parte de la UNAN-Managua, realiza el proceso de extensión con una diversidad de actividades tales como elaboración de inventarios de especies, diagnósticos de contaminación de aguas, recomendaciones de mejores prácticas a las industrias, educación ambiental a la población, elaboración de modelos arquitectónicos, evaluación de materiales, charlas y capacitaciones en reforzamiento de temas relacionados al comportamiento físico y químico de residuos, capacitaciones a estudiantes en astronomía y en la parte de computación creación de software y proyectos de evaluación y capacitación tecnológica. Es notorio que el desarrollo del proceso de extensión no es estándar en los diferentes departamentos académicos de la Facultad, se requiere de mayor atención en los departamentos de Biología y Tecnología ya que las carreras que ofrecen son adecuadas para establecer vínculo con el entorno y no se realiza en la actualidad.

El impacto de la extensión universitaria en pro de la mejora de la calidad de vida de la población nicaragüense es prioridad en la aplicación de conocimientos y técnicas en las carreras de la Facultad de Ciencias e Ingenierías.

\section{BIBLIOGRAFÍA}

Gonzalez, R. M. (7 de 02 de 2017). Quimica y Sociedad Nicaraguense. (J. d. Bonilla Anduray, Entrevistador)

Parrales Cano, H. (6 de Febrero de 2017). Extensión del Departamento de Matemáticas. (J. d. Bonilla Anduray, Entrevistador)

Pedroza Pacheco, M. E. (2015). Gestión de la Investigación, la Docencia y la Extensión Universitaria en la UNAN Managua. En M. E. Pedroza Pacheco, El Modelo de Gestión de la Investigación, Modelo I+D+i de la UNAN Managua (págs. 44-52). Managua: Editorial Universitaria.

Red mesoamericana de investigación y desarrollo de biocombustibles. (19 de Noviembre de 2016). Obtenido de Portal Oficial del Proyecto Mesoamérica: http://www.proyectomesoamerica. org/

UNAN Managua. (2015). Función Proyección y Extensión . En U. Managua, Plan Estratégico Institucional 2015-2019 (pág. 30). Managua: Editorial Universitaria . 